Négociations autour du cimetière musulman en Suisse : un exemple de recomposition religieuse en situation d'immigration

Sarah Burkhalter

\author{
(2) OpenEdition \\ Journals \\ Édition électronique \\ URL : http://journals.openedition.org/assr/20194 \\ DOI : 10.4000/assr.20194 \\ ISSN : $1777-5825$ \\ Éditeur \\ Éditions de l'EHESS
}

Édition imprimée

Date de publication : 1 avril 2001

Pagination : 133-148

ISBN : 2-222-96701-5

ISSN : 0335-5985

Référence électronique

Sarah Burkhalter, « Négociations autour du cimetière musulman en Suisse : un exemple de recomposition religieuse en situation d'immigration ", Archives de sciences sociales des religions [En ligne], 113 | janvier-mars 2001, mis en ligne le 19 août 2009, consulté le 20 avril 2019. URL : http:// journals.openedition.org/assr/20194; DOI : 10.4000/assr.20194

Ce document a été généré automatiquement le 20 avril 2019

(ㄷ) Archives de sciences sociales des religions 


\title{
Négociations autour du cimetière musulman en Suisse : un exemple de recomposition religieuse en situation d'immigration
}

\author{
Sarah Burkhalter
}

1 Depuis 1992, date de fermeture du "carré » musulman à Genève par les autorités, les musulmans de Suisse n'ont plus de lieu d'inhumation où ils puissent respecter les rites funéraires islamiques. Des négociations ont été entamées dans plusieurs cantons du pays pour tenter de remédier à cette situation. Quelles informations ces négociations nous donnent-elles sur l'attitude religieuse d'une communauté en situation d'immigration?

Dès 1992, on peut constater que l'activité déployée par la communauté musulmane pour trouver une solution permettant l'inhumation islamique en Suisse est considérable. D'autant plus que cette énergie est tout à fait nouvelle, si l'on considère que la question du cimetière musulman ne s'est posée pour la première fois qu'en 1978 à Genève, alors que le premier centre islamique datait déjà de 1961. Pourquoi ce regain d'intérêt pour organiser l'inhumation rituelle en Suisse et comment cette revendication s'exprime-telle?

\section{Développement d'une revendication}

3 À l'image des autres pays d'Europe, la communauté musulmane de Suisse s'est considérablement développée. Dans les années soixante, la plupart des immigrés étaient des chefs de famille venus travailler quelques années et amasser un peu d'argent avant de rentrer chez eux. Les immigrés, encore faibles en nombre, exprimaient peu de revendications vis-à-vis de la société d'accueil. La seule nécessité de l'époque était alors d'organiser des lieux de prière, sans structure socioculturelle particulière. Un cimetière musulman ne représentait donc pas une préoccupation majeure. La plupart des immigrés 
n'avaient pas l'intention de s'installer. Ils avaient maintenu la plus grande part de leurs liens avec le pays d'origine et préféraient être enterrés dans la terre des ancêtres. Ce n'est qu'avec le regroupement familial que la communauté a commencé à organiser plus largement sa vie dans le pays d'accueil, et à exprimer davantage de revendications quant à la place qu'elle voulait prendre dans cette société ${ }^{1}$.

D'autre part, cette population, à l'instar des autres populations étrangères, tend à se stabiliser. D'immigrés, de plus en plus d'entre eux deviennent résidents permanents. Dès lors, la motivation d'organiser sa vie dans la société d'accueil sur tous les plans, aussi bien culturels, sociaux que religieux, va en s'accroissant.

C'est ainsi que la question du cimetière est rapidement apparue comme centrale, à partir du moment où la population n'a plus pu profiter du «carré » musulman du PetitSaconnex à Genève. À Neuchâtel par exemple, un rapport du délégué aux étrangers affirme que "l'inexistence d'un cimetière musulman semble être le problème le plus important auquel la population musulmane est confrontée. » Cette inquiétude semble être largement partagée à Zurich, puisque les responsables musulmans ont cherché à entrer en négociations avec la Ville à peine une année après la fermeture du cimetière du Petit-Saconnex. D'autre part, on peut remarquer que l'ensemble des centres islamiques de la ville se sont rassemblés derrière ce projet, ce qui suppose une certaine unité quant aux revendications formulées. Le porte-parole de la Fondation culturelle islamique et mosquée du Petit-Saconnex, H .Ouardiri, porte-parole de la Fondation et mosquée du Petit-Saconnex à Genève, affirme que les deux priorités actuelles de la Fondation sont un espace pour une école islamique et un cimetière ou « carré » musulman. Enfin, les Suisses convertis à l'islam ont très vite exprimé leur volonté d'avoir des cimetières islamiques puisqu'une Fondation des cimetières islamiques suisses (F.C.I.S.) existe déjà depuis 1987, soit avant même la fermeture du cimetière du Petit-Saconnex. On constate donc une certaine unité dans la volonté de régler la question de l'inhumation rituelle en territoire suisse.

\section{Hétérogénéité de l'islam en Suisse}

Selon quels termes s'exprime la revendication actuelle du droit au cimetière musulman ? L'unité constatée quant à la volonté de régler la question du cimetière musulman se retrouve-t-elle dans les propositions faites pour réaliser ce projet? Quels renseignements ces attitudes nous donnent-elles quant à la recomposition du corpus religieux en situation d'émigration?

7 La population musulmane de notre pays se caractérise par une très grande hétérogénéité, que ce soit sur les plans linguistique (arabe, turc, yougoslave, etc.), national ou confessionnel $^{2}$. D'autres paramètres entrent en jeu, notamment le niveau socioéconomique et le niveau d'instruction. L'ancienneté de l'immigration et le régime politique et économique du pays d'origine peuvent également nous renseigner, par exemple pour comprendre l'attitude face au pays d'accueil et le niveau d' " engagement identitaire ». Ainsi, plus que le credo islamique, c'est la référence faite à la religion qui diffère beaucoup d'une frange de la population à l'autre. Malgré cette diversité, on se rend compte que la population musulmane de Suisse, toute appartenance nationale confondue, choisit massivement de rapatrier le corps dans le pays d'origine plutôt que de le faire enterrer sur le territoire suisse ${ }^{3}$. Nous avons choisi, dans cette étude, de nous 
intéresser spécialement aux responsables religieux, ou chefs de centres islamiques. Nous aurons donc l'occasion de voir comment ils interprètent ce recours au rapatriement, et quelle valeur religieuse ils y voient. Cela nous renseignera d'une part sur le discours et la position défendue par les responsables, et d'autre part sur l'écart qui réside entre cette position et la pratique de la majorité.

\section{Un discours unitaire}

Notre échantillon comprend une vingtaine de responsables de centres ou d'associations islamiques et d'imams de toute la Suisse, de nationalités et de niveaux d'instruction divers. Nous avons choisi de nous concentrer sur des responsables sunnites uniquement, ce qui en fait un échantillon relativement homogène. En effet, bien que le sunnisme comprenne quatre écoles de droit, cette diversité entre peu en ligne de compte dans le sujet qui nous intéresse ${ }^{4}$. Le choix de ne prendre en compte que des imams sunnites est légitime, dans la mesure où ce sont eux qui participent aux dialogues menés avec les autorités. Les shi'ites (très minoritaires) et les sufis, par exemple, s'ils n'ont pas les mêmes préoccupations vis-à-vis de la mort, ne le font pas savoir officiellement. L'homogénéité formelle de notre échantillon en fait son intérêt, puisque c'est justement cette homogénéité «de principe» que nous cherchons à vérifier. L'appartenance au sunnisme, et à une même corporation, suffit-elle pour présenter une unité de vues ? C'est ce que nous verrons. Le bagage personnel, le niveau d'éducation - qui diffère d'un imam à l'autre $\mathrm{s}^{5}$ et le pays de provenance, avec toutes les variantes socio-culturelles qui en découlent, entraînent des positions idéologiques variées et créent des divergences dans l'interprétation des textes religieux. Il se peut aussi qu'une certaine compétition existe entre différents centres religieux, ou que certains revendiquent une filiation spirituelle d'un cheikh particulier, lié au pays d'origine, ce qui pousserait les responsables à présenter des discours opposés. Or, à travers tous nos entretiens, on constate une singulière unité dans le discours vis-à-vis de la question du cimetière musulman en Suisse.

9 De manière quasi générale, ce discours tend à présenter la revendication du droit au cimetière comme étant de caractère purement religieux. Ainsi, l'évolution de la communauté musulmane en Suisse, sa progression numérique, et son intégration socioéconomique dans notre société n'auraient aucune part dans l'explication de la revendication actuelle. H.Ramadan, directeur du Centre islamique des Eaux-Vives à Genève, parle du droit à l'inhumation comme d'un droit religieux, faisant partie de la liberté de culte prônée par la Constitution suisse. Dans le même sens, il compare le cimetière à la mosquée, par la teneur religieuse de cet espace. À son avis, il faut considérer le cimetière comme un prolongement du lieu de culte, c'est-à-dire comme un espace sacré. Il nous semble que cette idée est partagée par la plupart de nos interlocuteurs, qui n'ont cessé de nous rappeler le caractère sacré du corps mort et l'impératif de respecter absolument son repos (interdiction d'exhumation ou de changement d'affectation du terrain). Cette soudaine distinction du sacré et du profane, peu présente dans l'islam, est-elle dictée par la situation de vie en immigration? On peut se poser la question. Toujours est-il que l'ensemble de l'argumentaire d'une majorité de nos interlocuteurs vise à démontrer le caractère strictement religieux du cimetière et de l'inhumation, sans considération aucune pour les aspects social, culturel ou économique. Seules trois personnes ont insisté sur l'importance sociale et culturelle du cimetière : un 
représentant de musulmans et musulmanes de Suisse (M.M.S.), organisation largement préoccupée par l'intégration de la deuxième génération des musulmans de Suisse, la fondatrice de l'Association culturelle des femmes musulmanes de Suisse, N.Karmous, et enfin un Suisse converti à l'islam et fondateur de la F.C.I.S., A .Meyers.

Il faut dès lors nous demander d'où vient cette homogénéité du discours. Pourquoi cette insistance sur le caractère religieux de la revendication? Que révèle-t-elle du rapport des responsables religieux avec le corpus?

11 Avant de traiter ces différentes questions, nous examinerons les motivations possibles de la revendication du droit au cimetière musulman. Ces motivations sont-elles d'ordre strictement religieux, comme l'affirment les responsables religieux?

\section{Les motivations de la revendication}

Quelles sont les motivations réelles des personnes interrogées dans leur revendication du droit au cimetière? Sur un plan théorique, les motivations que nous avons isolées sont d'ordre religieux, culturel ou socio-politique. Selon nos propres définitions, la motivation religieuse est la volonté stricte de respecter une foi et un rituel ; la motivation culturelle est celle qui s'attache de manière plus affective et plus large à des pratiques traditionnelles, moins par souci religieux que par désir de respecter la coutume et de perpétuer des traditions ; la motivation socio-politique est la volonté de reconnaissance et d'intégration sociale et politique. Cette distinction a surtout une valeur méthodologique, puisque la réalité est plus complexe ${ }^{6}$. Voyons maintenant si les points revendiqués par les responsables musulmans sont tous « religieux » comme ils le disent.

Si la demande de cimetières musulmans est vraiment de type religieux, c'est donc qu'il y a une impossibilité à respecter le rite funéraire islamique dans l'inhumation "à la ligne » des cimetières communaux. Voyons ce qu'il en est. Le principal problème de l'inhumation sur sol suisse est qu'elle ne permet pas de respecter le repos perpétuel des défunts. C'est là le seul obstacle majeur à l'application du rituel, puisque si nous nous en tenons aux obligations de base, nous pouvons admettre qu'il est possible de les respecter. Les Hadiths parlent en effet de trois obligations dans le rituel funéraire : la toilette du mort, la mise en terre, le visage tourné vers la Mecque, et les prières sur le mort. Pour ce qui est de la toilette du corps, il est de la liberté de chacun de l'effectuer selon son rite; elle est donc faite par l'imam ou un proche du défunt, conformément au rituel islamique. Quant à la mise en terre, en direction de la Mecque, plusieurs informateurs, que ce soit du côté musulman ou de celui des Pompes funèbres, nous ont assuré que les fossoyeurs acceptaient volontiers de décaler le corps par rapport à l'arrangement extérieur de la tombe pour respecter la direction voulue. Enfin, les prières sur le mort ne posent aucun problème quant à leur application, et peuvent avoir lieu soit à la mosquée, soit au cimetière.

14 Le plus gênant serait donc l'impossibilité d'avoir des tombes perpétuelles, et la peur de l'exhumation. En ce qui concerne l'exhumation, il ne semble pas que ce soit la question qui motive la communauté de Zurich dans sa revendication, puisque P. Wittwer nous a assuré que les corps chrétiens n'y étaient pas non plus exhumés. Le système des tombes à étages est pratiqué. Après le quatrième ou cinquième étage, on s'arrête et on laisse les ossements en terre. Or, que ce soit à Zurich ou à Neuchâtel, les représentants des communautés musulmanes ont admis la solution des tombes à étages dans les cimetières 
musulmans, ce qui veut dire qu'ils acceptent de rouvrir une fosse pour y déposer un nouveau corps après un certain temps, ou du moins de superposer plusieurs tombes dans un espace relativement restreint ${ }^{7}$. Le problème peut survenir au moment où le cimetière est désaffecté pour être transformé par exemple en parc public, sans que les corps soient déterrés et seulement après une durée de 150 ou 200 ans. Nos interlocuteurs musulmans ont bien insisté sur le fait que le cimetière ne devait pas servir de lieu de promenade et qu'il était impensable de marcher sur une tombe. Cette idée est aussi présente dans les Hadiths. En voici un exemple rapporté par A .D .Al-Djazaïri ${ }^{8}$ : «Il vaut mieux pour l'un de vous de s'asseoir sur une braise ardente qui brûle ses habits jusqu'à atteindre sa peau, que de s'asseoir sur un tombeau (Moslim)». A .D .Al-Djazaïri en conclut : « Le musulman ne doit pas s'asseoir sur un tombeau, ni le fouler des pieds. »

Outre la peur de l'exhumation, la dispersion des tombes musulmanes dans les cimetières communaux nous a été présentée par l'ensemble des personnes interrogées comme le principal problème «religieux » de l'inhumation en Suisse. La volonté de séparation des morts musulmans d'avec les autres est présentée comme un impératif majeur par la F.C.I.S.

Nos différents interlocuteurs ont également beaucoup insisté sur ce fait comme étant une obligation religieuse. Cette volonté de séparation peut être comprise comme religieuse dans le sens où les docteurs de la loi prescrivent la séparation des tombes. Examinons maintenant quelles sont les raisons données pour rassembler les tombes musulmanes. Nos interlocuteurs ont tous mentionné l'existence d'une relation spirituelle entre les vivants et les morts. Y. Basalamah nous a précisé que cette relation n'existait pas seulement au niveau de la famille, et c'est cela qui est important, mais de la communauté tout entière. $\mathrm{H}$.Ouardiri explique : « un musulman ne visite pas seulement la tombe de sa famille, mais aussi celles des autres ». Relation qui, plus qu'un culte du souvenir, semble prendre la forme d'une sorte de transmission spirituelle d'une génération à l'autre. Idée qui rappelle les différents cultes populaires de saints qui s'organisent dans nombre de pays arabo-musulmans, et qui sont régulièrement combattus par les tenants de l'orthodoxie'.

17 Nous pensons que cette motivation relève davantage du domaine culturel ou affectif que du domaine purement religieux. Nos interlocuteurs, tout en répétant par ailleurs que cette séparation des corps était obligatoire, ont davantage utilisé un vocabulaire de type affectif que religieux. Il nous a, par exemple, souvent été répété qu'il était «normal d'avoir envie » d'être enterré au sein de la communauté avec laquelle on avait vécu toute sa vie durant. $\mathrm{H}$.Ramadan nous a affirmé : « Nous sommes une communauté jusque dans l'au-delà ». Les tombes musulmanes n'ont d'ailleurs pas besoin d'être absolument isolées des tombes chrétiennes. Aux dires de nos interlocuteurs, il semble que l'idée de rassemblement des tombes musulmanes prime sur le fait d'être séparé des chrétiens. À Zurich comme à Neuchâtel, les délégués musulmans chargés de négocier avec l'État sur la question du cimetière ont admis, voire montré une nette préférence pour la solution du partage des cimetières communaux ${ }^{10}$.

D'où vient cette affirmation? À première vue, la notion d'Umma, la Communauté des croyants, occupe une place centrale dans l'islam qui s'est développé au cours des siècles, et non forcément à la période prophétique. Les islamologues répètent souvent que l'islam est din wa dawla, religion et cité. C'est dire que la vie communautaire constituerait à elle seule un des deux principes de base de l'islam. L.Gardet ${ }^{11}$ écrit: " l'islam est à la fois religion et communauté temporelle; mieux encore: une communauté qui prend en 
charge en un seul et indissociable élan les relations de chaque croyant avec Dieu, et les relations des croyants les uns avec les autres sur le plan moral, social, politique. » D'autre part, si la séparation des tombes ne fait l'objet d'aucune prescription coranique ou sunnique (de la Sunna), les écoles juridiques ont admis par la suite, et selon les règles de l'interprétation coranique, que cette séparation devait avoir lieu. La question est de savoir quelle définition nous donnons à la religion. Une religion n'est-elle formée que des Textes sacrés, ou inclut-elle également les divers commentaires élaborés selon des règles théologiques précises et véhiculant un certain nombre de valeurs et de principes développés dans l'esprit de ces Textes?

L'union des croyants serait donc au cœur de l'islam, mais non en tant que prescription coranique. Elle l'est davantage au titre de coutume, de volonté de « former corps » autour d'une même croyance. Cette exigence relève en effet moins du rite (prescrit par un texte) que de la pratique (geste découlant d'une croyance ou d'une valeur religieuse). Reste que lorsque nos interlocuteurs utilisent le mot "religieux", ils se réfèrent avant tout à l'ensemble formé par le Coran et la Sunna (Hadiths). En présentant alors l'exigence de séparation des corps comme une obligation religieuse, ils étendent le champ du religieux à un domaine qu'eux-mêmes ne considèrent pas comme tel dans leur discours. Nous reviendrons plus loin sur les raisons et la signification de cette attitude. L'insistance mise sur le lien entre les croyants devant la mort est d'autant plus frappante que celle-ci revêt un caractère individuel dans l'islam coranique (le musulman est seul devant la mort, jugé pour ses propres actes). Ainsi, le prolongement de l'existence de la communauté au-delà de la mort est artificiel, construit. Il semble donc que ce souci d'assurer une unité à la communauté est surtout motivé par des impératifs d'ordre culturel (préservation d'une culture) et socio-politique (nécessité de survie ou volonté de perpétuation). Cette volonté de distinction existe naturellement dans toute nouvelle religion, au moment où il lui faut se distinguer de celles qui existent déjà. Il est impressionnant de voir que ce besoin de légitimité perdure au-delà des siècles. Comme si le contexte d'immigration représentait une nouvelle menace, justifiant une telle volonté de démarcation, ou de visibilité.

Notre interprétation culturelle ou socio-politique des motivations réelles de nos interlocuteurs est encore renforcée par un autre élément. Le fait qu'une part grandissante de la population musulmane - relayée par les responsables religieux émette le vœu d'être enterrée sur territoire suisse, serait révélateur, sinon d'un début d'intégration socioculturelle réelle dans la société d'adoption, du moins du désir de cette intégration.

21 Le recours actuel au rapatriement des corps dans le pays d'origine exprime en effet un attachement affectif ou culturel au pays d'origine. Le fait d'être enterré avec sa famille, dans la terre des ancêtres, ne constitue pas une obligation religieuse, ni même une solution préférée par l'islam. C.Yassine ${ }^{12}$ parle de la peur de rompre la filiation avec le père par le choix d'un autre lieu d'inhumation que la terre des ancêtres. C'est alors par souci de respecter l'ordre généalogique qu'on se fait enterrer dans le caveau familial. L'appartenance est ici culturelle, et le sentiment d'identité plus national ou culturel que religieux. Ainsi, le fait même qu'on accepte d'être enterré dans le pays d'accueil est la marque d'une meilleure intégration culturelle et sociale dans la société d'adoption. Cette volonté d'insertion est très forte chez les jeunes arabophones de Suisse romande. $P$.Haenni ${ }^{13}$ l'a vérifié. « Nombre de jeunes ont opté pour une insertion individuelle dans la société d'accueil en parallèle, ou à titre de substitut, aux liens de parenté au sein de la famille nucléaire ou élargie (soit parce qu'ils sont en position de démarcation à l'égard de 
leurs parents, soit parce qu'ils sont venus seuls, pour les études ou à la recherche de travail).»

De plus en plus, et à mesure que la population se sédentarise, les références culturelles pré-migratoires sont abandonnées pour faire place à une recomposition identitaire, même si celle-ci ne se fait pas selon un seul schéma. Par exemple, le porte-parole de M.M.S. a insisté sur le fait que les musulmans de deuxième génération ne se considèrent pas comme une communauté étrangère mais participant à la société suisse. Il a précisé que la deuxième génération avait davantage de racines en Suisse que dans son pays d'origine.

La revendication du droit au cimetière s'inscrit dans cette perspective d'intégration socio-politique de la communauté musulmane. T. Facchinetti, délégué aux étrangers du canton de Neuchâtel, observe d'ailleurs une certaine « cristallisation de ce problème de la reconnaissance sociale et politique sur la question du cimetière ", qui lui apparaît surtout comme un enjeu symbolique, bien que légitime. Ce souci d'intégration, nous l'avons ressenti fortement tout au long de nos entretiens. Non comme une volonté de se fondre complètement dans la société, au sens d'assimilation, mais plutôt comme le désir d'être reconnu avec son identité propre. À cet égard, nombre de plaintes nous ont été formulées, traduisant cette préoccupation. Plusieurs interlocuteurs ont déploré : «Ils ne veulent pas de nous, même morts». Ou encore: "Si on trouve de la place pour les vivants, on doit aussi en trouver pour les morts». Mme N. Karmous, fondatrice de l'Association culturelle des femmes musulmanes de Suisse, lie essentiellement la question du cimetière à celle de l'intégration sociale : «C'est un problème pour l'intégration de ne pas avoir de cimetière. Les musulmans le sentent comme un rejet. Même morts, ils ne se sentent pas acceptés. La réexpédition du corps est souvent une souffrance, car on se sépare du défunt d'une manière artificielle ». Une manière de dire que si la Suisse était vraiment prête à intégrer les vivants, alors elle montrerait plus de souplesse pour accepter les morts. H. Ouardiri, ne parle même plus d'intégration mais de citoyenneté : «La situation des musulmans n'est plus temporaire ici. Il faut penser cela en termes de citoyenneté, et non plus d'intégration ». Un discours qui exprime bien une volonté sociale et politique d'intégration et de reconnaissance d'une communauté musulmane établie, citoyenne de la Suisse.

\section{Références au religieux et réaménagements du corpus}

24 L'analyse des enjeux de la revendication du droit au cimetière pour la communauté musulmane de Suisse nous donne l'occasion d'observer un cas réel de réaménagement du corpus religieux en situation d'émigration. B. Lewis ${ }^{14}$ explique que la situation d'émigration volontaire actuelle est tout à fait nouvelle dans l'histoire musulmane. «À aucun moment, on a imaginé une situation où un musulman quitterait volontairement une terre musulmane pour se mettre dans une situation aussi délicate ». Jusqu'ici, les docteurs avaient discuté des cas de séjours provisoires des voyageurs ou ambassadeurs, ou ceux des prisonniers de guerre capturés par les incroyants. Or la catégorie des migrants volontaires est aujourd'hui nouvelle, et les attitudes à adopter envers les sociétés non-musulmanes sont peu discutées. La question du réaménagement du corpus est donc laissée la plupart du temps aux soins des responsables des centres islamiques, qui vont devoir gérer leur rapport au religieux et négocier eux-mêmes avec les autorités la place qu'ils vont occuper dans la société d'accueil. À notre connaissance, les ouvrages 
écrits par des musulmans et traitant de la vie musulmane dans les sociétés européennes sont très peu nombreux. Celui de $\mathrm{T}$.Ramadan ${ }^{15}$, personnalité musulmane engagée dans la société helvétique, Les musulmans dans la laïcité, est davantage qu'un manuel de conduite, c'est un code moral général, discutant des principes plus que de problèmes concrets. La question de l'inhumation n'y est par exemple pas traitée. Certains ouvrages traitent plus spécifiquement de la mort, mais sans prendre en compte du tout la question de la façon de vivre cet événement en situation d'immigration. Il semble donc que ce soit le rôle des intellectuels musulmans d'aujourd'hui de formuler les termes des compromis nécessaires entre application de la foi et participation aux principes laïcs des sociétés occidentales. Plusieurs auteurs affirment ces réaménagements possibles. D .Schnapper ${ }^{16}$ rappelle par exemple qu'en cas de nécessité (darura), il est prévu par le Coran qu'une obligation soit abandonnée ou un interdit enfreint. Reste à savoir si les conditions de vie en terre nonmusulmane sont considérées comme des cas de nécessité. J.-P .Charnay ${ }^{17}$ le dit également: "l'islam admet la variabilité de ses prescriptions en fonction de la contingence au moins en certaines limites et sous certaines conditions. » Rappelons que ces réaménagements du rituel sont prévus déjà dans les Hadiths. En ce qui concerne le rituel funéraire, il y est dit, par exemple, qu'en cas de guerre ou d'hécatombe, il est possible d'enterrer les corps deux à deux, chose que l'on préfère éviter en temps normal. La juridiction islamique comporte plusieurs outils d'interprétation de la loi. La consultation ou concertation (shura), le consensus (ijma') et l'effort d'interprétation personnelle (ijtihad) donnent cette possibilité de former des Conseils de sages pouvant délibérer à tout moment lorsqu'un problème d'application de la loi se pose. Reste à savoir s'ils sont utilisés, comment ils le sont et par qui. Si l'islam admet une lecture dynamique de la loi, cela ne veut pas dire que la communauté sache en profiter. D'autre part, il faudrait également pouvoir établir dans quelle mesure l'interprétation personnelle est possible. Selon $\mathrm{M}$.Arkoun ${ }^{18}$, elle ne peut se faire qu'à partir d'un certain nombre de postulats de base qu'elle doit respecter.

Que pouvons-nous dire de ces réaménagements dans le cas du cimetière musulman en Suisse ? Constate-t-on une réélaboration du rituel ? En quoi cette réélaboration consistet-elle? Nous reprendrons ces questions plus loin en rapport avec notre terrain, lorsque nous vérifierons si les différentes attitudes observées vis-à-vis du corpus religieux sont motivées par la situation de vie en immigration ou non.

\section{Réaménagements au niveau du rite}

Les trois obligations de base (toilette, inhumation, prières), bien qu'elles soient présentées dans le discours comme étant les trois seules obligatoires, n'apparaissent pas suffisantes ni à la majorité des croyants, ni aux responsables musulmans, pour que ceuxci aient l'impression de respecter le rituel funéraire. Dit plus simplement, l'importance réelle n'est pas seulement attribuée aux principaux points du rite funéraire. La popularité de certains rites ne correspond pas à l'importance dont ils font l'objet dans les textes religieux. Par exemple, le caractère impératif donné à la séparation des corps musulmans d'avec les morts d'autres confessions représente selon nous une surévaluation de cet élément du rituel. Certes, c'est là le point nodal de la revendication du droit au cimetière, puisque la séparation des cimetières est fondée principalement sur la séparation des corps. Mais ce n'est pas non plus le seul argument existant pour exiger des espaces distincts. D'autres points du rite, comme la direction de la Mecque ou l'éternité du repos 
des morts devraient constituer des impératifs tout aussi importants. D'ailleurs, nos interlocuteurs ne les ont pas évacués. L'importance donnée à la nécessité de regrouper les tombes musulmanes n'apparait disproportionnée que lorsqu'on la compare avec son degré d'obligation «légal». Non seulement cette pratique ne fait l'objet d'aucune obligation coranique ou traditionnelle (Hadiths), mais elle n'est même pas conseillée ou suggérée dans les Textes. Elle n'apparaît qu'au niveau des développements juridiques des siècles postérieurs à la Sunna.

Cela représente-t-il une réinterprétation abusive du rituel funéraire? Non, dans le sens où l'esprit de la loi suggère que la communauté des croyants est unie et que cette union est un reflet de celle que la communauté entretient avec Dieu ; pas non plus, dans le sens où la tradition (Hadiths) affirme nettement un lien véritable entre les vivants et les morts, et que ce lien ne peut mieux s'exprimer que par des prières sur les tombes rassemblées des défunts de la communauté. Par là, nous voyons que le rituel entretient un rapport complexe avec les Textes, et cela vaut de manière générale. Par exemple, les Hadiths ou le Coran ne disent pas non plus expressément que le repos du mort doit être éternel, mais cette idée découle logiquement des enseignements que le Coran ou les Hadiths donnent sur l'au-delà. C'est l'affirmation coranique de la résurrection des corps qui justifie la nécessité du repos perpétuel du défunt. De même, c'est l'affirmation de l'union des croyants de l'Umma et la réalité du lien entre les vivants et les morts qui justifient le regroupement des tombes musulmanes. Certains commentateurs ont certainement encouragé cette idée selon laquelle l'islam invite à une séparation des corps par confession. Al-Ghazali ${ }^{19}$, par exemple, dans son traité d'eschatologie La Perle précieuse, rapporte une histoire qui devait vraisemblablement se transmettre à son époque : "On raconte encore d'un Arabe du désert qu'il dit à son fils : «Qu'est-ce que Dieu t'a fait [une fois mort]?» Son fils répondit : «Il ne m'a pas fait de mal, mais je suis enterré dans le voisinage d'un certain homme qui fut un impie, et je suis tout effrayé de voir quels châtiments il doit subir." " S'il ne s'agit ni d'un ajout dans le rituel ni d'une interprétation abusive, on peut pourtant parler d'un rapport particulier au religieux, qui tend à élargir son champ au maximum. Nous reviendrons plus loin sur cette idée.

Qu'est-ce qui motive ce réajustement du rituel ? Nous pensons que l'insistance mise sur la séparation des corps représente typiquement une attitude dictée par une situation particulière : l'immigration. Nous ne voulons pas dire par là que la séparation des corps musulmans ne se fait pas dans les cimetières des pays musulmans, mais que l'importance donnée à cet élément du rite est caractéristique d'une situation d'immigration. Nous pensons en effet que c'est le besoin de regroupement d'une communauté minoritaire, et la nécessité de l'unité des différents membres de cette communauté (même morts) qui sont à l'origine de cette insistance. Alors qu'on comprend facilement que le besoin de s'unifier soit caractéristique d'une communauté qui vit en situation minoritaire dans un pays étranger, le besoin de réaffirmer le lien entre les vivants et les morts semble moins évident. Il procède pourtant, selon nous, du même phénomène. Rappelons rapidement que les visites sur les tombes ne se font pas à la seule famille mais à la communauté tout entière, de même que les prières d'intercession sont adressées à tous les morts du cimetière. On comprend dès lors que rassembler les morts signifie réaffirmer une solidarité intracommunautaire. La peur de disperser ses morts signifie donc aussi une crainte de voir les membres de la communauté tomber dans un individualisme égoïste qui les éloignerait les uns des autres. Il nous semble que ce besoin de solidarité et d'identité collective est dicté en grande partie par la situation d'immigration que vit la communauté 
musulmane. Ces réajustements du religieux sont donc aussi stratégies de survie en terre non-musulmane. Nous comprenons donc à quel point l'attachement à ce rite n'est pas seulement religieux mais aussi culturel ou social.

L'inadéquation entre les niveaux de popularité de certains rites et leur place dans le canon représente-t-elle une incohérence de la part des chefs religieux? Si ceux-ci accordent la plus grande importance au respect de la forme la plus officielle du rituel, le fait qu'ils insistent sur des points à première vue mineurs du rituel signifie-t-il une incohérence ou un manque de sincérité dans leur discours? Nous pensons que l'attitude consistant à élargir le champ religieux ou à insister davantage sur un élément du rituel dénote une certaine difficulté de la part des responsables religieux à délimiter le champ de leur religion. Cette attitude peut également être comprise comme le fait de ne pas aborder la religion d'un point de vue uniquement littéral ou textuel. La signification ou l'esprit du rite comporte autant, voire davantage, d'importance aux yeux des hommes de religion.

\section{Rapport au corpus religieux}

30 À partir du regard porté sur le rituel funéraire, nous avons cru pouvoir dégager une attitude générale de nos interlocuteurs vis-à-vis du corpus religieux. Nous avons en effet constaté une attitude qui favorise, du moins dans le discours, un islam débarrassé des ajouts ou des scories pré-islamiques relevant de la coutume. Cette attitude est-elle vraiment suivie d'applications? Plusieurs responsables nous ont fait part de leur choix de renoncer aux coutumes des fidèles qui fréquentent leurs centres. A .Bihi, responsable d'un centre islamique à Lausanne, nous a par exemple appris que beaucoup de ses membres venaient de pays noirs-africains, dont les traditions populaires étaient particulièrement fortes, et n'avaient souvent rien à voir avec l'islam lui-même. Il avait préféré interdire ces pratiques coutumières. Il ne faut pas y voir forcément une preuve de fondamentalisme ou la manifestation d'une orthodoxie exagérée, mais peut-être simplement le souci de préserver une religion dans sa forme originale, préoccupation qui peut encore être renforcée dans un contexte d'immigration. La préservation d'un islam coranique représente donc peut-être aussi une stratégie de survie. Par conséquent, ces réaménagements de la foi peuvent être aussi compris comme des réactions face à l'environnement. Réactions nécessaires à la préservation de la religion, ou à la protection d'une identité. Quand l'exil se prolonge, le risque d'assimilation augmente, ce qui pousse à la reconstruction d'une identité collective forte, fondée si possible sur une vision commune de sa tradition et non sur une disparité de points de vue. D'où peut-être ce souci d'assurer un islam le plus cohérent et unitaire possible de la part des dirigeants musulmans. Nous ne disons pas que cette vision unitaire existe, mais qu'elle est recherchée par les responsables musulmans. En effet, face à ce souci de maintenir l'islam à un niveau purement religieux, nous constatons certaines incohérences dans l'attitude de nos interlocuteurs. Par exemple, l'insistance mise sur l'unité de la communauté audelà de la mort, et qui justifie à leurs yeux la nécessité de rassembler les tombes musulmanes, ne s'appuie pas directement sur le corpus religieux. Cette insistance relève autant de la culture ou d'une stratégie de survie sociale que du respect de la religion. Et pourtant, elle est présentée dans le discours comme une exigence purement religieuse par la plupart des personnes interrogées. De même, l'attitude consistant à définir l'espace du cimetière comme sacré alors que l'islam coranique ne distingue pas le sacré du profane 
présente elle aussi une contradiction. À quoi sont dues ces incohérences? N’oublions pas que si le fait de se référer à un islam coranique est généralement partagé par tous les imams et responsables de centres que nous avons rencontrés, cela ne signifie pas forcément qu'ils soient d'accord sur le contenu de cet islam. On peut alors se demander si le corpus religieux existe vraiment sous une forme unique. Les Hadiths eux-mêmes ne donnent pas une version unique du même rituel et beaucoup de points de détail sont eux aussi soumis à des variations. Cette polymorphie du rituel islamique donne donc lieu à une multitude d'interprétations possibles. Il est normal, dès lors, que les imams aient des visions différentes sur la manière de respecter au mieux la forme officielle d'un rite. D'autre part, le corpus religieux est également caractérisé par un certain flou. Où s'arrête et où commence le religieux? Comment déterminer un rituel qui serait purement religieux ? Faut-il considérer comme faisant partie du corpus les rituels découlant des interprétations juridiques des siècles postprophétiques?

31 Cette recherche d'un islam coranique est-il uniquement dicté par la situation d'immigration ? Ne relève-t-il pas aussi d'un choix délibéré ? Il est certain que la diversité d'origine des membres de chaque centre (nous parlons ici surtout des centres arabophones, et non des turcophones) rend impossible l'application des pratiques coutumières. Y .Ibram, directeur d'un centre islamique à Zurich, nous a, lui aussi, expliqué que les pays d'origine des membres de son centre étaient trop nombreux pour qu'il soit imaginable de prendre en compte les traditions de chacun. Il a donc choisi d'appliquer un islam coranique, parce que commun à tous. Il est clair que le meilleur moyen d'unir une communauté autour d'une identité est d'assurer une certaine unité de ce bagage identitaire. On a donc bien, de la part des responsables religieux eux-mêmes, une volonté d'assurer coûte que coûte une unité de la communauté, un rassemblement des membres autour d'une même pratique, d'une même compréhension des textes religieux. Cette volonté d'unité et de solidarité, si elle existe naturellement dans toute confession, est certainement exacerbée par les conditions de vie en immigration et par la peur de voir la communauté se dissoudre au sein de la société dominante.

\section{Attachement à un islam purement religieux}

Cette insistance sur un islam purement religieux et débarrassé de ses éléments culturels peut être mise en rapport avec la présentation avant tout religieuse de la question du cimetière musulman en Suisse. En effet, on peut comprendre cette insistance comme une volonté de marginalisation des autres arguments, non islamiques et donc non légitimes. Ce qui expliquerait pourquoi les responsables musulmans sous-estiment volontairement les motivations culturelles ou socio-politiques de leur revendication. Laissons un moment de côté la question des causes (situation d'immigration ou autre) de cette attitude. Ce qui nous semble intéressant pour l'instant c'est de mettre en parallèle l'attitude consistant à ne prendre en considération que l'argument religieux et la volonté de l'isoler. L'un et l'autre procèderaient d'un même mouvement, à savoir, la volonté de placer le religieux au centre du cadre identitaire. Voici un texte qui confirme notre analyse. Parlant des sociétés musulmanes, A.Sayad ${ }^{20}$ montre qu'une longue histoire de domination «a imprimé à la religion et à la relation au religieux, aussi bien de la part des nonmusulmans (i.e. des dominants) que de la part des musulmans eux-mêmes (i.e. des dominés), un profil de religion dominée parce que religion de sociétés dominées, et a fait que partout, dans toutes ces sociétés, l'identité qu'on dirait sociale, culturelle, voire 
politique est devenue, en réaction à la domination des autres, inséparable de l'identité religieuse, celle-ci n'étant alors, et souvent, que l'expression la plus autorisée de celle-là ; la plus autorisée parce qu'elle se couvre de l'autorité de la religion et s'autorise de la sacralisation qu'elle s'est elle-même conférée.»

Cette insistance sur le religieux peut également donner lieu à un phénomène d'empiètement du religieux sur les autres sphères de la pensée. Nous en avons eu une illustration avec l'exigence de séparation des tombes. Cette attitude consiste à étendre au maximum le champ du religieux, pour englober l'ensemble des commentaires juridiques développés dans les siècles post-prophétiques. Ainsi, dans certains cas, les responsables musulmans insistent sur l'importance de limiter le religieux au Coran (islam coranique), alors qu'à d'autres moments ils insistent sur le caractère religieux d'un élément qui n'est présent que dans la jurisprudence (figh) et non dans la Loi elle-même (charia). Cette attitude, à première vue paradoxale, s'explique par la volonté de placer le religieux au centre de l'argumentation. Dans le premier cas, nous avons une définition étroite du religieux ; dans le deuxième cas, une définition la plus large possible.

Cette volonté d'insister sur le religieux est certainement dictée par les conditions de vie en immigration, et les besoins de présenter une revendication la plus légitime possible. Or, qu'est-ce qui légitime une revendication issue de la communauté musulmane, si ce n'est des impératifs islamiques? Seules des obligations religieuses peuvent justifier des privilèges accordés à un groupe se revendiquant comme communauté religieuse.

\section{Conclusion}

L'étude de la revendication du droit au cimetière musulman en Suisse nous a permis d'observer un exemple de réajustement religieux en contexte d'immigration. L'inévitable dialogue avec les autorités du pays d'accueil, la confrontation avec les lois existantes, entrainent forcément une relecture de ses traditions et de ses croyances. Dans le cas présent, nous avons constaté une certaine surévaluation de l'argument religieux de la revendication du droit au cimetière. À l'intérieur de cette attitude, nous avons cru trouver un paradoxe, puisque parallèlement à la volonté de dépouiller le religieux de ses éléments extérieurs, nous avons observé une tendance à élargir le plus possible le champ du religieux. Cette attitude se révèle moins paradoxale si nous considérons le but ultime de nos interlocuteurs, qui serait de placer le religieux au centre de l'identité de la communauté et de l'argumentation afin de légitimer la revendication.

La demande de cimetières séparés n'est pas une volonté d'isolement par rapport à la société d'accueil, mais un désir d'y être intégré, avec son identité propre. Le mouvement de visibilité religieuse actuel révèle une volonté d'organiser sa vie ici, en refusant de s'y sentir éternellement étranger. Nous partageons en cela la conclusion de P.Haenni ${ }^{21}:$ « Ni la demande, ni l'offre d'islam qui se dessine en Suisse francophone ne se destine fondamentalement au " développement séparé » (même si, certes, des tendances dans ce sens existent), mais insistent sur la nécessité d'intervenir sur la scène publique et sur le terrain social ». D'ailleurs, la réapparition de revendications de type religieux ne signifie pas forcément une crispation sur une identité passéiste. Au contraire, la volonté d'insertion s'accompagne, nous l'avons vu, d'une réaffirmation d'une identité propre. Bien que ce double mouvement puisse paraître paradoxal, il ne l'est pas forcément : c'est au moment où l'on se rend compte qu'on est en train de s'intégrer que des velléités 
d'affirmation identitaire réapparaissent, comme si, maintenant qu'on était suffisamment intégré pour être accepté et se défendre, on pouvait enfin se permettre de réaffirmer ce qu'on avait préféré cacher au moment où l'on se sentait si différent. Cette réaffirmation n'est d'ailleurs la plupart du temps pas un simple retour aux sources, mais recomposition. Nous concluons avec T .Ramadan ${ }^{22}$ : «Quand des individus ou des associations de «la communauté musulmane" interpellent les pouvoirs publics en vue de trouver des solutions aux divers problèmes qui sont les leurs, ils ne traduisent pas une volonté d'être traités différemment; bien plutôt - puisqu'ils vont vivre ici - ils demandent à ce qu'on prenne en considération leur présence et leur identité dans le cadre d'une législation qui a été élaborée en leur absence. » Pouvoir vivre selon sa foi, c'est là la liberté recherchée.

\section{BIBLIOGRAPHIE}

AL-BOKHARI Muhammad Ibn Isma'il, Les traditions islamiques, trad. O. Houdas et W. Marais, t. I, Paris, Maisonneuve, 1977.

AL-GHAZALI, La Perle précieuse, trad. L. Gautier, Paris, Londres, Maisonneuve \& Cie, Williams \& Norgate, 1878 .

AL-DJAZAïRI Aboubaker Djaber, La voie du musulman, trad. M. Chakroun, Paris, Aslim éd., 1986.

ARKOUN Mohammed, GARDET Louis, L'Islam hier-demain, Paris, Buchet/Chastel, 1978.

CHARNAY Jean-Paul, Sociologie religieuse de l'Islam, (1978) Paris, Hachette, 1994.

GARDET Louis, L'Islam, religion et communauté, Paris, Desclée de Brouwer, 1970.

HAENNI Patrick, « Dynamiques sociales et rapport à l'État. L'institutionnalisation de l'Islam en Suisse », in Revue Européenne des Migrations Internationales, vol. 10, n 1, 1994, pp. 183-198.

HAENNI Patrick, « Musulmans de Suisse et religion : d'un Islam à l'autre », in Minorités chrétiennes et musulmans. Aspects religieux, Actes de colloque de déc. 1993, coordonné par J. Waardenburg, Université de Lausanne, Cahiers du DIHSR, n 4, 1995, pp. 8-47.

HAJA Fawzia, La mort et le Jugement Dernier selon les enseignements de l'Islam, s.l. (France), Arayhane éd., 1991.

La population musulmane du canton de Neuchâtel, rapport du bureau du délégué aux étrangers,

Y. von KAENEL, 1996.

LEWIS B., SCHNAPPER Dominique, eds. Musulmans en Europe, Poitiers, Actes Sud, 1992.

RAMADAN Tarik, Les musulmans dans la laïcité. Responsabilités et droits des musulmans dans les sociétés occidentales, Lyon, Tawhid, 1994.

SAYAD Abdelmalek, « Minorités et rapport à l'État dans le monde méditerranéen », in Connaissance de l'islam, (coll.), Paris, Syros, 1992, pp. 135-181.

YASSINE Chaïb, «Pour une thanatologie maghrébine : les rapatriements de corps », in Annuaire de l'Afrique du Nord, vol. XXVII, 1988, pp. 337-348. 


\section{NOTES}

1. De 1970 à 1990, le nombre total des musulmans en Suisse a presque décuplé. On est passé d'une population de 16353 en 1970 à 152217 en 1990. Lors du dernier recensement, la population musulmane représentait donc $2,2 \%$ de la population totale, et $12 \%$ de la population étrangère.

2. Les ressortissants musulmans proviennent d'une quarantaine de pays différents; les communautés les plus importantes sont celles de Turquie $(45,1 \%$ de la population musulmane étrangère), relativement ancienne, et de Yougoslavie (38,4\%), plus récente. La communauté turque connaît elle-même une forte hétérogénéité, confessionnelle (sunnites, shi'ites, alawites), politique (socialistes, nationalistes...), ou ethnique (Turcs, Kurdes).

3. Les chiffres sont difficiles à obtenir, puisque les centres eux-mêmes ne tiennent pas de registres indiquant qui se fait rapatrier et qui enterrer en Suisse. Mais différents regroupements d'informations nous permettent d'affirmer que le rapatriement est largement préféré.

4. Les quatre écoles (shafa'ite, malikite, hanafite et hanbalite) ne diffèrent pratiquement pas quant au rituel funéraire. Les différents imams et cheikhs interrogés nous ont certifié que seule la manière d'accomplir la prière sur le mort variait d'une école à l'autre.

5. Il n'est en effet pas nécessaire d'avoir suivi toute la filière universitaire islamique pour être chef de centre en Suisse. Certains de nos interlocuteurs étaient donc docteurs, alors que d'autres n'avaient suivi que l'école coranique.

6. Par exemple, une référence au corpus religieux peut être faite dans un esprit d'appartenance culturelle et vice versa.

7. Ceci représente une marque d'ouverture de la part des responsables musulmans, quand on sait que les Hadiths interdisent généralement d'ouvrir une tombe pour y placer un deuxième corps ou d'enterrer deux corps dans la même tombe, sauf en cas de nécessité (hécatombe, guerre). Toutefois, les pays arabo-musulmans utilisent les tombeaux de famille, ce qui signifie une accumulation de corps dans le même espace.

8. AL-DJAZAïrI, 1986, p. 301.

9. En effet, l'islam coranique interdit le culte des morts, même celui du Prophète lui-même, et limite de façon très précise les durées de deuil, au-delà desquelles le souvenir du mort ne doit en aucun cas envahir le quotidien des survivants ni les empêcher de continuer à vivre.

10. Partage d'un même cimetière en plusieurs « carrés » ou zones.

11. GARDET, 1970, p. 273.

12. YASSINE, 1988, p. 338.

13. HAENNI, 1995, p. 29.

14. LEWIS, SCHNAPPER, 1992, p. 32.

15. RAMADAN, 1994.

16. LEWIS, SCHNAPPER, op. cit., p. 182.

17. CHARNAY, 1994, p. 312.

18. ARKOUN, GARDET, 1978, p. 169.

19. Al-GHAZALI, 1878, p. 25.

20. SAYAD. 1992, p. 148.

21. HAENNI, op. cit., p. 32.

22. RAMADAN, op. cit., p. 97. 


\section{RÉSUMÉS}

La communauté musulmane de Suisse est privée de cimetière musulman depuis 1992, date de fermeture du carré musulman au cimetière du Petit-Saconnex à Genève. Des négociations ont alors rapidement vu le jour entre les différentes autorités cantonales et les responsables religieux musulmans. Cette étude se penche sur le discours présenté par les chefs de centres islamiques et responsables religieux qui participent de près ou de loin à ces débats. Alors que les entretiens montrent une étonnante unité dans le discours, qui tend à présenter cette revendication du droit au cimetière comme principalement religieuse, une étude plus approfondie des exigences posées par les responsables au cours des négociations mettent en lumière des préoccupations culturelles et socio-politiques, qui viennent se greffer sur les arguments religieux de la revendication. Cette tendance à centrer le dialogue sur l'élément religieux reflète une recherche de légitimité de la part des responsables musulmans. Seuls des impératifs religieux peuvent en effet légitimer une telle revendication auprès des autorités de la société d'accueil.

The muslim community of Switzerland is deprived from cemetery since 1992, when the muslim patch in Geneva's cemetery has been closed. Therefore, several negocia-tions has been initiated by the religious leaders with the different local authorities. This study tries to analyse the position presented by the religious leaders who take part in these negociations with the government. Though the interviews show a surprising unity in the discourse of the leaders, who present the claim like a purely religious one, a more acute analyse of the points required by them in the negociations lighten cultural and socio-political motivations, which come to complete the religious ones. This tendency to centre the dialogue on the religious motivation denote a quest of legitimity from the religious leaders. As a matter of fact, only religious obligations can give legitimation to such a claim within the negocations with the hostsociety's authorities.

La comunidad musulmana de Suiza ha sido privada de cementerio desde el año 1992 ; año en el que la parte musulmana del cementerio de Ginebra fue cerrada. A consecuencia de esto los líderes religiosos iniciaron distintas negociaciones con diferentes autoridades locales. Este estudio intenta analizar las propuestas de los líderes religiosos que toman parte en estas negociaciones con el gobierno. Las entrevistas muestran una sorprendente unidad en el discurso de estos líderes, quienes presentan sus reivindicaciones como puramente religiosas. A pesar de esto, un análisis más agudo de los puntos tratados, requiere, por parte de ellos, un esclarecimiento de las motivaciones culturales y sociopolíticas que aclare la base religiosa de su discurso en las negociaciones. Esta tendencia a centrar el diálogo en los motivos religiosos denota una búsqueda de legitimidad por parte de los líderes religiosos. Y es que, a decir verdad, sólo las obligaciones religiosas pueden legitimar estas reivindicaciones en el marco de las negociaciones con las autoridades de la sociedad de acogida. 